Algebraic $8 \mathcal{G}$ Geometric Topology

Volume 3 (2003) 709-718

Published: 30 July 2003

ATG

\title{
Fixed point data of finite groups acting on 3-manifolds
}

\author{
PÉTER E. Frenkel
}

\begin{abstract}
We consider fully effective orientation-preserving smooth actions of a given finite group $G$ on smooth, closed, oriented 3-manifolds $M$. We investigate the relations that necessarily hold between the numbers of fixed points of various non-cyclic subgroups. In Section 2, we show that all such relations are in fact equations mod 2 , and we explain how the number of independent equations yields information concerning low-dimensional equivariant cobordism groups. Moreover, we restate a theorem of A. Szücs asserting that under the conditions imposed on a smooth action of $G$ on $M$ as above, the number of $G$-orbits of points $x \in M$ with non-cyclic stabilizer $G_{x}$ is even, and we prove the result by using arguments of G. Moussong. In Sections 3 and 4, we determine all the equations for non-cyclic subgroups $G$ of $S O(3)$.
\end{abstract}

AMS Classification 57S17; 57R85

Keywords 3-manifold, group action, fixed points, equivariant cobordism

\section{Preliminaries}

We shall use the word "representation" to mean a representation of a group by means of proper rotations of a three-dimensional Euclidean linear space.

Let $H$ be a subgroup of the group $G$, and let $\rho$ be an equivalence class of representations of $H$. Denote by $N_{G}(H, \rho)$ the subgroup of $G$ formed by those elements $g \in G$ whose conjugation action fixes $(H, \rho)$ (that is, $g$ normalizes $H$ and the conjugation action leaves the equivalence class $\rho$ invariant).

We shall use the word "manifold" to mean a smooth, closed, oriented threedimensional manifold (if not otherwise stated). All group actions shall be assumed smooth and orientation-preserving. Consider an action $G \rightarrow \operatorname{Diff}^{+}(M)$ of the finite group $G$ on the manifold $M$. Suppose that no nontrivial element of $G$ acts via the identity map on any component of $M$. (We shall refer to 
this assumption by saying that the action of $G$ is "fully effective". If $M$ is connected, then "fully effective" is the same as "effective".)

Choose a $G$-invariant Riemannian metric on $M$. Then the fixed point set $M^{g}$ of an arbitrary element $1 \neq g \in G$ is a finite disjoint union of closed geodesics without self-intersections. For $x \in M^{g}$, the proper orthogonal transformation $T_{x} g \in S O\left(T_{x} M\right)$ is a rotation of finite order about the axis $T_{x} M^{g}$. It follows that if $M^{g}$ and $M^{h}$ have a common component, then $g$ and $h$ generate a cyclic subgroup of $G$. Therefore, the fixed point set $M^{H}$ of any non-cyclic subgroup $H$ of $G$ is finite. So is the set $Q$ of all points with non-cyclic stabilizer.

For a non-cyclic subgroup $H$ of $G$, denote by $n^{H}$ the number of $N_{G}(H)$-orbits of points $x \in M$ whose stabilizer $G_{x}$ is exactly $H$. This is the number of such points divided by the index $\left|N_{G}(H): H\right|$. In other words, $n^{H}$ is the number of $G$-orbits of points $x \in M$ whose stabilizer $G_{x}$ is a conjugate of $H$.

For any subgroup $H$ of $G$ and equivalence class $\rho$ of faithful representations of $H$, denote by $M_{\rho}^{H}$ the set of points $x \in M^{H}$ such that the induced representation of $H$ on $T_{x} M$ is of the equivalence class $\rho$. For non-cyclic $H$, denote by $n_{\rho}^{H}$ the number of $N_{G}(H, \rho)$-orbits of points $x \in M_{\rho}^{H}$ such that $G_{x}=H$ (these shall be called "points of type $(H, \rho)$ "). This is the number of such points divided by the index $\left|N_{G}(H, \rho): H\right|$. In other words, $n_{\rho}^{H}$ is the number of $G$-orbits of points $x \in M$ whose stabilizer $G_{x}$ together with its induced representation on $T_{x} M$ is a conjugate of $(H, \rho)$.

We obviously have $n^{H}=\sum_{\rho} n_{\rho}^{H}$, where $\rho$ runs over those faithful representations of $H$ that are inequivalent even if conjugation by elements of $N_{G}(H)$ is allowed. We also have $n_{\rho}^{H}=n_{\rho^{\prime}}^{H^{\prime}}$ if $(H, \rho)$ and $\left(H^{\prime}, \rho^{\prime}\right)$ are equivalent under the conjugation action of $G$.

Note that the sets $M^{H}$ and $M_{\rho}^{H}$ and the numbers $n^{H}$ and $n_{\rho}^{H}$ do not depend on the $G$-invariant metric that we have chosen.

\section{Arbitrary groups}

For any finite group $G$, consider the additive Abelian group $\mathcal{A}=\mathcal{A}(G)$ of all integer-valued $G$-invariant functions defined on the set of pairs $(H, \rho)$ with $H$ a non-cyclic subgroup of $G$ and $\rho$ an equivalence class of faithful representations of $H$. We shall be concerned with the set $\mathcal{F}_{+}=\mathcal{F}_{+}(G)$ of those functions in $\mathcal{A}(G)$ that are realizable as the function $n$ associated to a fully effective action of $G$ on a manifold. We define $\mathcal{F}=\mathcal{F}(G)$ as the additive subgroup of $\mathcal{A}(G)$ generated by $\mathcal{F}_{+}(G)$. 
Remark 2.1 Any $n \in \mathcal{F}_{+}(G)$ is realizable by an effective action of $G$ on a connected manifold.

Proof Consider a realization on a manifold $M$. If $M$ is not connected, then choose points $x$ and $y$ in different components with trivial stabilizer. Delete small neighborhoods that correspond to the open 3 -ball of radius $1 / 2$ under a diffeomorphism of a greater neighborhood onto the open 3-ball of radius 1 . Identify the boundaries of the deleted neighborhoods in a way compatible with the given orientations. Do the same for $g(x)$ and $g(y)(g \in G)$, using the $g-$ image of the neighborhoods used for $x$ and $y$. The resulting manifold admits a fully effective $G$-action with the same numbers $n_{\rho}^{H}$, and has fewer components than $M$ does. Iterating this procedure, we arrive at a connected manifold.

Lemma 2.2 (i) $\mathcal{F}_{+}$is the set of non-negative functions in the additive group $\mathcal{F}$.

(ii) $\mathcal{F} \geq 2 \mathcal{A}$.

Proof (i) The action of $G$ on $G \times M$ via left translations on $G$ shows that $0 \in \mathcal{F}_{+}$. Taking disjoint union of manifolds shows that $\mathcal{F}_{+}$is closed under addition.

Suppose that $n, n^{\prime} \in \mathcal{F}_{+}$and $n^{\prime} \geq n$. We prove that $n^{\prime}-n \in \mathcal{F}_{+}$. To this end, choose fully effective actions of $G$ on manifolds $M$ and $M^{\prime}$ that give rise to $n$ and $n^{\prime}$, respectively. A suitable $G$-invariant neighborhood of $Q \subset M$ (the set of points with non-cyclic stabilizer), consisting of small open balls, is $G-$ equivariantly diffeomorphic to a $G$-invariant neighborhood of a $G$-invariant subset of $Q^{\prime} \subset M^{\prime}$. The diffeomorphism can be chosen to be orientationreversing, because central reflection in the origin of $\mathbb{R}^{3}$ commutes with any proper rotation of $\mathbb{R}^{3}$ and reverses orientation. Delete both neighborhoods and identify their boundaries to get a closed manifold endowed with a fully effective $G$-action that proves that $n^{\prime}-n \in \mathcal{F}_{+}$.

(ii) We first construct a fully effective action of $G$ on a manifold $M$ that has $n_{\rho}^{H}>0$ for an arbitrarily chosen non-cyclic subgroup $H$ of $G$ and an arbitrarily chosen faithful representation $\rho$ of $H$. To this end, we let the subgroup $H$ act on $S^{3}=\mathbb{R}^{3} \cup\{\infty\}$ by rotations of $\mathbb{R}^{3}$ given by the representation $\rho$, and we form the twisted product $M=G \underset{H}{\times} S^{3}$.

Choose a point $x \in M$ of type $(H, \rho)$. Take two copies of $M$, and delete the two copies of a neighborhood of $Q \backslash G x \subset M$, consisting of small open balls. 
Identify their boundaries in a $G$-equivariant and orientation-reversing way to get a closed manifold endowed with a fully effective $G$-action that has $n_{\rho}^{H}=2$, and has $n_{\rho^{\prime}}^{H^{\prime}}=0$ if $\left(H^{\prime}, \rho^{\prime}\right)$ is not equivalent to $(H, \rho)$ under the conjugation action of $G$.

András Szücs called my attention to the fact that the quotient groups $\mathcal{A} / \mathcal{F}$ and $\mathcal{F} / 2 \mathcal{A}$ can be interpreted in terms of low-dimensional equivariant cobordism groups. These interpretations are given in the following two propositions. We do not use them in the sequel. The proofs are left to the reader.

An action of $G$ on a manifold with boundary is called quasi-free if all stabilizers are cyclic subgroups of $G$.

Proposition 2.3 The quotient group $\mathcal{A}(G) / \mathcal{F}(G)$ is the quasi-free cobordism group of equivariantly (but not necessarily quasi-freely) nullcobordant closed oriented two-dimensional manifolds endowed with fully effective $G$-actions.

Note that if $\mathcal{A}(G) \neq 0$ (that is, if $G$ has a subgroup isomorphic to a non-cyclic subgroup of $S O(3)$ ), then $\mathcal{A}(G) / \mathcal{F}(G) \neq 0$. This shall be seen in Corollary 2.6.

Let $\Omega_{3}(G)$ stand for the oriented equivariant cobordism group of closed 3manifolds with free $G$-actions, and let $\hat{\Omega}_{3}(G)$ stand for the oriented equivariant cobordism group of those with arbitrary fully effective $G$-actions.

Proposition 2.4 The sequence

$$
\Omega_{3}(G) \rightarrow \hat{\Omega}_{3}(G) \rightarrow \mathcal{F}(G) / 2 \mathcal{A}(G) \rightarrow 0
$$

is exact.

I do not know if it is true that the map on the left is always zero, so that $\hat{\Omega}_{3}(G)=\mathcal{F}(G) / 2 \mathcal{A}(G)$ (cf. [2, Corollary 3.4], [3], [4], and [5] for results of this kind in the unoriented case). At any rate, the dimension of the $\mathbb{F}_{2}$-linear space $\hat{\Omega}_{3}(G) / 2 \hat{\Omega}_{3}(G)$ is bounded from below by that of $\mathcal{F}(G) / 2 \mathcal{A}(G)$.

The following theorem of A. Szücs imposes a relation on the values of any $n \in \mathcal{F}(G)$, showing in particular that $\mathcal{F}(G) \neq \mathcal{A}(G)$ if $\mathcal{A}(G) \neq 0$. The theorem will prove helpful in Section 4 when we deal with the octahedral group.

Theorem 2.5 Let $G$ act fully effectively on $M$. Then the set $Q$ of those points $x \in M$ that have a non-cyclic stabilizer consists of an even number of $G$-orbits. 
The following proof is due to G. Moussong.

Proof Let $P$ stand for the set of points in $M$ whose stabilizer is non-trivial. Then $P$ is a graph (one-dimensional complex) whose set of vertices (zerodimensional skeleton) is $Q$. The set $P \subset M$ is $G$-invariant, and if $g \in G$ fixes an interior point of an edge of $P$, then it fixes each point of that edge. Therefore, $P / G$ is a graph whose set of vertices is $Q / G$.

Choose any point $x \in Q$. The degree in $P / G$ of the vertex $G x \in Q / G$ is the number of $G_{x}$-orbits of directed axes of the rotations given by the faithful representation $G_{x} \longmapsto S O\left(T_{x} M\right)$. This number is always 3, as is well known from the classification of finite non-cyclic subgroups of $S O(3)$. So the graph $P / G$ is $3-$ regular, and therefore it has an even number of vertices.

Corollary 2.6 Let $n \in \mathcal{F}(G)$. Then the sum $\sum n_{\rho}^{H}$, with $(H, \rho)$ running over all $G$-inequivalent pairs, is even.

Note that if $n^{G} \neq 0$ for some $n \in \mathcal{F}(G)$, then $G$ is (isomorphic to) a finite noncyclic subgroup of $S O(3)$. We shall explicitly describe $\mathcal{F}(G)$ (or, equivalently, $\left.\mathcal{F}_{+}(G)\right)$ for these groups $G$ in Section 3 and Section 4. T. tom Dieck [1, Theorem 6] solved a similar problem for the group $Z_{2}^{k}$ acting on an unoriented manifold of arbitrary dimension. That investigation deals only with the fixed points of the entire group though.

\section{Dihedral groups}

In this section, we explicitly describe $\mathcal{F}$ for the dihedral group $D_{m}$.

For $m>2$, there are exactly $\varphi(m) / 2$ inequivalent faithful representations of the cyclic group $Z_{m}$. Each extends in a unique way to a faithful representation of the dihedral group $D_{m}$. The two-element group $Z_{2}$ and the Kleinian group $D_{2}$ have unique faithful representations (up to equivalence).

The subgroups of the dihedral group $D_{m}$ are dihedral groups themselves. For each $k$ dividing $m$, there are $m / k$ subgroups of isomorphism type $D_{k}$. If $m / k$ is odd, then each one is its own normalizer and they are all conjugates of each other. If $m / k$ is even, then each one has a $D_{2 k}$ as its normalizer and they form two classes that shall be marked by $\triangle$ and $\nabla$. For any representatives of the two classes, $D_{k}^{\triangle} \cap D_{k}^{\nabla}=Z_{k}$. So a function $n \in \mathcal{A}\left(D_{m}\right)$ is given by its values $n_{\rho}^{D_{k}}$ (for $m / k$ odd and $\rho$ a faithful representation of $Z_{k}$ ), and $n_{\rho}^{D_{k}^{\triangle}}$ and $n_{\rho}^{D_{k}^{\nabla}}$ (for $m / k$ even and $\rho$ a faithful representation of $Z_{k}$ ). 
Theorem 3.1 Let $m \geq 2$. The function $n \in \mathcal{A}\left(D_{m}\right)$ is in $\mathcal{F}\left(D_{m}\right)$ if and only if the following two conditions hold for all $k \geq 2$ dividing $m$.

(i) If $m / k$ is odd, then $n_{\rho}^{D_{k}}$ is even for all $\rho$.

(ii) If $m / k$ is even, then $n_{\rho}^{D_{k}^{\Delta}}$ and $n_{\rho}^{D_{k}^{\nabla}}$ have the same parity for all $\rho$.

Proof First suppose that $n \in \mathcal{F}\left(D_{m}\right)$. We prove that (i) and (ii) hold. We may assume that $n \in \mathcal{F}_{+}\left(D_{m}\right)$. Let $n$ be realized by a fully effective action on $M$.

(i) The claim is equivalent to saying that there is an even number of points of type $\left(D_{k}, \rho\right)$ for any subgroup of isomorphism type $D_{k}$ and for any $\rho$. We shall prove that the number of such points on any component $S^{1}$ of $M_{\rho}^{Z_{k}}$ is either two or zero. Suppose that the point $x \in S^{1}$ is of type $\left(D_{k}, \rho\right)$. Consider the action of the two-element quotient group $D_{k} / Z_{k}$ on $S^{1}$. The non-trivial element fixes $x$ and reverses orientation on $S^{1}$. Therefore, it has a unique fixed point on $S^{1}$ besides $x$, which is also of type $\left(D_{k}, \rho\right)$.

(ii) Suppose to the contrary that, say, $n_{\rho}^{D_{k}^{\triangle}}$ is odd and $n_{\rho}^{D_{k}^{\nabla}}$ is even. Using Lemma 2.2, we may assume that $n_{\rho}^{D_{k}^{\Delta}}=1$ and $n_{\rho}^{D_{k}^{\nabla}}=0$. This means that, for any representatives of the two classes, there are no points of type $\left(D_{k}^{\nabla}, \rho\right)$ and there are exactly two points, say $x$ and $y$, of type $\left(D_{k}^{\triangle}, \rho\right)$. The argument that we have used to prove (i) shows that the points $x$ and $y$ must be on the same component $S^{1}$ of $M_{\rho}^{Z_{k}} \backslash M^{Z_{2 k}}$. For the $D_{2 k}$ subgroup generated by the chosen $D_{k}^{\triangle}$ and $D_{k}^{\nabla}$ subgroups, the coset $D_{2 k} \backslash D_{k}^{\triangle}$ must interchange $x$ and $y$. So $D_{2 k}$ must leave $S^{1}$ invariant. The Kleinian quotient group $D_{2 k} / Z_{k}$ acts on $S^{1}$. The coset $Z_{2 k} \backslash Z_{k}$ does not have fixed points on $S^{1}$, so it preserves orientation on $S^{1}$. The subgroup $D_{k}^{\triangle}$ does have fixed points on $S^{1}$, so the coset $D_{k}^{\triangle} \backslash Z_{k}$ reverses orientation on $S^{1}$. Therefore, the coset

$$
D_{k}^{\nabla} \backslash Z_{k}=\left(Z_{2 k} \backslash Z_{k}\right)\left(D_{k}^{\triangle} \backslash Z_{k}\right)
$$

also reverses orientation and must have two fixed points. These must be of type $\left(D_{k}^{\nabla}, \rho\right)$, which is a contradiction.

To prove the converse, choose any $k$ dividing $m$, and choose a faithful representation $\rho$ of $Z_{k}$. Pick a generator $g$ in $Z_{m}$ for which the generator $g^{m / k}$ of $Z_{k}$ is represented by a rotation through $2 \pi / k$. Let $g$ act on $S^{3} \subset \mathbb{C}^{2}$ by

$$
g(z, w)=\left(e^{2 \pi i k / m} z, e^{2 \pi i / m} w\right) .
$$


Choose an arbitrary element of the coset $D_{m} \backslash Z_{m}$ and let it act by complex conjugation on both coordinates. This defines an action of $D_{m}$ on $S^{3}$. The set of points whose stabilizer non-trivially intersects $Z_{m}$ is the circle

$$
S^{1}=\{w=0\}=M_{\rho}^{Z_{k}} .
$$

All points $x$ of $S^{1}$ have $G_{x} \cap Z_{m}=Z_{k}$, so $G_{x}$ is either $Z_{k}$ or a $D_{k}$ subgroup. The two-element quotient group $D_{k} / Z_{k}$ of each $D_{k}$ subgroup acts on $S^{1}$. The coset $D_{k} \backslash Z_{k}$ reverses orientation on $S^{1}$ and therefore has exactly two fixed points. If $m / k$ is even, then this means that $n_{\rho}^{D_{k}^{\triangle}}=n_{\rho}^{D_{k}^{\nabla}}=1$ and all other numbers $n_{\rho}^{H}$ are zero.

The theorem now follows from Lemma 2.2.

Corollary 3.2 (i) The dimension of the $\mathbb{F}_{2}$-linear space $\mathcal{A}\left(D_{m}\right) / \mathcal{F}\left(D_{m}\right)$ is $\lfloor m / 2\rfloor$.

(ii) The dimension of the $\mathbb{F}_{2}$-linear space $\mathcal{F}\left(D_{m}\right) / 2 \mathcal{A}\left(D_{m}\right)$ is $\lfloor m / 4\rfloor$ if $m$ is even and is zero if $m$ is odd.

Proof (i) The dimension is the total number of inequivalent faithful representations of the cyclic groups $Z_{k}$ with $k \geq 2$ dividing $m$, which is

$$
\sum_{2<k \mid m} \varphi(k) / 2 \quad \text { resp. } \quad 1+\sum_{2<k \mid m} \varphi(k) / 2
$$

as $m$ is odd or even. The claim follows from the fact that $\sum_{k \mid m} \varphi(k) / 2=m / 2$ and $\varphi(1)=\varphi(2)=1$.

(ii) The dimension is the total number of inequivalent faithful representations of the cyclic groups $Z_{k}$ with $k \geq 2$ and $m / k$ even. This equals zero for odd $m$ and equals $\lfloor(m / 2) / 2\rfloor=\lfloor m / 4\rfloor$ for even $m$.

\section{Groups of rotations of the Platonic solids}

In this section, we explicitly describe $\mathcal{F}$ for the tetrahedral, the octahedral, and the icosahedral group. We shall frequently use the expression "a conjugation action of $G$ on $S O(3)$ ", which shall mean an injective homomorphism $G \longmapsto$ $S O(3)$ composed with the conjugation action $S O(3) \longmapsto \operatorname{Diff}^{+}(S O(3))$ of $S O(3)$ on $S O(3)$. Note that a suitable diffeomorphism between $S O(3)$ and $\mathbb{R} P^{3}$ turns this into an action of $G$ on $\mathbb{R} P^{3}=\mathbb{R}^{3} \cup \mathbb{R} P^{2}$ by rotations of $\mathbb{R}^{3}$ extended to $\mathbb{R} P^{3}$. 
The only non-cyclic proper subgroup of the tetrahedral group $A_{4}$ is the Kleinian group $D_{2}$. Faithful representations of both $A_{4}$ and $D_{2}$ are unique up to equivalence. So a function $n \in \mathcal{A}\left(A_{4}\right)$ is given by its two values $n^{A_{4}}$ and $n^{D_{2}}$.

Theorem 4.1 The function $n \in \mathcal{A}\left(A_{4}\right)$ is in $\mathcal{F}\left(A_{4}\right)$ if and only if $n^{D_{2}}$ and $n^{A_{4}}$ are of the same parity.

Proof "Only if" is a particular case of Corollary 2.6. It also follows from Theorem 3.1(i) when applied to $k=m=2$.

To prove the converse, observe that $n^{A_{4}}=1$ holds for the conjugation action of $A_{4}$ on $S O(3)$. The theorem now follows from Lemma 2.2.

Corollary 4.2 (i) $\operatorname{dim}_{\mathbb{F}_{2}} \mathcal{A}\left(A_{4}\right) / \mathcal{F}\left(A_{4}\right)=1$.

(ii) $\operatorname{dim}_{\mathbb{F}_{2}} \mathcal{F}\left(A_{4}\right) / 2 \mathcal{A}\left(A_{4}\right)=1$.

The non-cyclic subgroups of the octahedral group $S_{4}$ are of isomorphism type $S_{4}, A_{4}, D_{4}, D_{3}$ and $D_{2}$. All have unique faithful representations. Any two of them that are isomorphic are conjugates of each other, except for one of the four Kleinians which is normal in $S_{4}$ and shall be denoted by $D_{2}^{*}$. So a function $n \in \mathcal{A}\left(S_{4}\right)$ is given by its six values $n^{S_{4}}, n^{A_{4}}, n^{D_{4}}, n^{D_{3}}, n^{D_{2}}$ and $n^{D_{2}^{*}}$.

Theorem 4.3 The function $n \in \mathcal{A}\left(S_{4}\right)$ is in $\mathcal{F}\left(A_{4}\right)$ if and only if the four numbers $n^{D_{3}}, n^{D_{4}}, n^{S_{4}}$ and $n^{D_{2}^{*}}+n^{D_{2}}+n^{A_{4}}$ are of the same parity.

Proof To prove "only if", suppose that $n \in \mathcal{F}_{+}\left(S_{4}\right)$ is realized by an action of $S_{4}$ on a manifold. Restriction of that action to a $D_{3}$ subgroup shows that $n^{D_{3}}+n^{S_{4}} \in \mathcal{F}_{+}\left(D_{3}\right)$. Restriction to a $D_{4}$ subgroup shows that $n^{D_{4}}+n^{S_{4}}$ is the value at $D_{4}$ of a function in $\mathcal{F}\left(D_{4}\right)$. Theorem 3.1(i), when applied to $k=m=3$ and to $k=m=4$, shows that $n^{D_{3}}, n^{D_{4}}$ and $n^{S_{4}}$ are of the same parity. Corollary 2.6 says that

$$
n^{D_{3}}+n^{D_{4}}+n^{S_{4}}+n^{D_{2}^{*}}+n^{D_{2}}+n^{A_{4}}
$$

is even, so "only if" is proved.

To prove the converse, consider the conjugation actions of the subgroups $H=$ $S_{4}, A_{4}$ and $D_{4}$ on $S O(3)$, and form twisted products $S_{4} \underset{H}{\times S O}(3)$. The functions 
$n$ associated to these three actions of $S_{4}$ are linearly independent mod 2, since they take the values listed below at $S_{4}, A_{4}$ and $D_{2}$.

$\begin{array}{cccc} & S O(3) & S_{4} \times \underset{A_{4}}{\times} S O(3) & S_{4} \underset{D_{4}}{\times} S O(3) \\ S_{4} & 1 & 0 & 0 \\ A_{4} & * & 1 & 0 \\ D_{2} & * & * & 1\end{array}$

The theorem now follows from Lemma 2.2.

Corollary 4.4 (i) $\operatorname{dim}_{\mathbb{F}_{2}} \mathcal{A}\left(S_{4}\right) / \mathcal{F}\left(S_{4}\right)=3$.

(ii) $\operatorname{dim}_{\mathbb{F}_{2}} \mathcal{F}\left(S_{4}\right) / 2 \mathcal{A}\left(S_{4}\right)=3$.

The non-cyclic proper subgroups of the icosahedral group $A_{5}$ are of isomorphism type $A_{4}, D_{5}, D_{3}$ and $D_{2}$. Any two of them that are isomorphic are conjugates of each other. The icosahedral group has exactly two inequivalent faithful representations. We shall denote them by + and - . Their restriction to any of the six $D_{5}$ subgroups yields the two $(=\varphi(5) / 2)$ inequivalent faithful representations of that subgroup. These shall also be denoted by + and - , respectively. The faithful representations of the other non-cyclic subgroups are unique up to equivalence. So a function $n \in \mathcal{A}\left(A_{5}\right)$ is given by its seven values $n_{+}^{A_{5}}, n_{-}^{A_{5}}, n^{A_{4}}, n_{+}^{D_{5}}, n_{-}^{D_{5}}, n^{D_{3}}$ and $n^{D_{2}}$.

Theorem 4.5 The function $n \in \mathcal{A}\left(A_{5}\right)$ is in $\mathcal{F}\left(A_{5}\right)$ if and only if the four sums

$$
\begin{aligned}
& n^{D_{2}}+n^{A_{4}} \\
& +n_{+}^{A_{5}}+n_{-}^{A_{5}}, \\
& n^{D_{3}}+n_{+}^{A_{5}}+n_{-}^{A_{5}}, \\
& n_{+}^{D_{5}}{ }_{n_{-}^{D_{5}}}+n_{+}^{A_{5}}+n_{-}^{A_{5}}
\end{aligned}
$$

are even.

Proof "Only if" follows from Theorem 3.1(i) when applied to $k=m=2,3$, 5 and 5 , respectively.

To prove the converse, consider the two conjugation actions of $A_{5}$ on $S O(3)$. Also consider the conjugation action of an $A_{4}$ subgroup on $S O(3)$, and form the twisted product $A_{5} \underset{A_{4}}{\times} S O(3)$. The functions $n$ associated to these three 
actions of $A_{5}$ are linearly independent $\bmod 2$, since they take the values listed below at $\left(A_{5},+\right),\left(A_{5},-\right)$ and $A_{4}$.

$\begin{array}{cccc} & S O(3) & S O(3) & A_{5} \times \underset{A_{4}}{\times} S O(3) \\ \left(A_{5},+\right) & 1 & 0 & 0 \\ \left(A_{5},-\right) & 0 & 1 & 0 \\ A_{4} & 0 & 0 & 1\end{array}$

The theorem now follows from Lemma 2.2.

Corollary 4.6 (i) $\operatorname{dim}_{\mathbb{F}_{2}} \mathcal{A}\left(A_{5}\right) / \mathcal{F}\left(A_{5}\right)=4$.

(ii) $\operatorname{dim}_{\mathbb{F}_{2}} \mathcal{F}\left(A_{5}\right) / 2 \mathcal{A}\left(A_{5}\right)=3$.

\section{Acknowledgements}

I am deeply indebted to Professor András Szücs for calling my attention to the problem, and for helpful discussions. I am also grateful to Professor András Szenes for useful comments.

This research was partially supported by OTKA grant T-042769.

\section{References}

[1] T tom Dieck, Characteristic numbers of $G$-manifolds I, Invent. Math. 13 (1971) 213-224.

[2] S S Khare, Finite group action and equivariant bordism, Pacific J. Math. 116 (1985), no. 1, 39-44.

[3] Czes Kosniowski, Some equivariant bordism theories vanish, Math Annalen 242 (1979), 59-68.

[4] Czes Kosniowski, Fixed points and group actions, Algebraic topology, Aarhus 1982, 603-609, Lecture Notes in Math. 1051, Springer, Berlin, 1984

[5] RE Stong, Equivalent bordism and $Z_{2}^{k}$ actions, Duke Math. J. 37 (1970), $779-785$.

Department of Geometry, Mathematics Institute

Budapest University of Technology and Economics, Egry J. u. 1.

1111 Budapest, Hungary

Email: frenkelp@renyi.hu

Received: 7 January 2003

Algebraic 6 Geometric Topology, Volume 3 (2003) 